Revue d'histoire de l'Amérique française

ZRS REVUE D.HISTOIRE DE L'AMÉRIQUE FRANÇAISE

\title{
Un inventaire descriptif des documents de la session, 1841-1936
}

\section{Christian Pouyez}

Volume 31, numéro 4, mars 1978

URI : https://id.erudit.org/iderudit/303652ar

DOI : https://doi.org/10.7202/303652ar

Aller au sommaire du numéro

Éditeur(s)

Institut d'histoire de l'Amérique française

ISSN

0035-2357 (imprimé)

1492-1383 (numérique)

Découvrir la revue

Citer cette note

Pouyez, C. (1978). Un inventaire descriptif des documents de la session,

1841-1936. Revue d'histoire de l'Amérique française, 31(4), 563-574.

https://doi.org/10.7202/303652ar d'utilisation que vous pouvez consulter en ligne.

https://apropos.erudit.org/fr/usagers/politique-dutilisation/ 
NOTE DE RECHERCHE

\title{
UN INVENTAIRE DESCRIPTIF DES DOCUMENTS DE LA SESSION, 1841-1936*
}

\author{
Christian PouYez \\ Département des Sciences humaines \\ Université du Québec à Chicoutimi
}

Nous présentons, dans cette note de recherche, un projet ayant pour objectif la constitution d'un inventaire descriptif général des Documents de la Session du Québec. Soulignons tout de suite qu'il s'agit d'un travail d'équipe, auquel ont été associés Mlles Andrée Bouchard, Louise Tremblay, Ghislaine Neill, Andrée Duguay, et MM. Christian Dessureault, Jacques Ouellet et Rémi Grenon, tous étudiants au module d'Histoire de l'Université du Québec à Chicoutimi.

$$
-\mathrm{I}-
$$

Depuis les débuts du régime parlementaire, les gouvernements du Québec et du Canada publient régulièrement une série de rapports, d'études et de documents relatifs à tous les aspects de l'administration du pays: rapports annuels des ministères, comptes publics, statistiques diverses, correspondances, etc... De 1792 à 1859 inclusivement, ces documents sont publiés sous la forme d'Appendices aux Journaux de l'Assemblée Législative; à partir de 1860, ils constituent une publication autonome, que l'on désigne alors sous le

* Cette recherche a été financée par le Fonds institutionnel de Recherche et par le décanat des études avancées et de la recherche de l'Université du Québec à Chicoutimi. Nous les remercions sincèrement. 
titre de: Documents de la Session. Noter cependant que les Journaux de l'Assemblée contiennent encore, après 1860, des Appendices, en général assez brefs et en nombre très restreint. En 1867, la série des Documents de la Session se divise, le gouvernement fédéral publiant désormais ses propres documents, et les gouvernements provinciaux faisant de même. Au Québec, la série des Documents de la Session commence effectivement en 1869; il n'y a pas, en effet de Documents correspondant à la session 1867$1868^{1}$. La série se poursuit, sans interruption, jusqu'en 1936 inclusivement; à partir de 1915, les documents ne sont plus numérotés, et à compter de 1924, la série change de nom et s'intitule: Documents Parlementaires.

L'inventaire que nous avons entrepris couvre uniquement les Appendices aux Journaux de l'Assemblée Législative et les Documents de la Session publiés par le gouvernement du Canada de 1841 à 1866 et par le gouvernement du Québec de 1867 à 1936 (pour la simplicité de l'exposé, nous désignerons désormais l'ensemble de ces documents du terme: Documents de la Session). Indispensable à l'historien du Québec et du Canada, cette série documentaire demeure cependant d'un accès difficile: ampleur considérable de la collection (environ 80 pieds linéaires pour 1841-1866 et 1867-1936), absence d'instruments de repérage adéquats, tels que tables des matières ou index ${ }^{2}$ et volumes non paginés. Chaque volume des Documents de la Session est un assemblage plus ou moins hétéroclite de documents, dont quelques-uns peuvent être paginés, et qui ont parfois leur index particulier, mais il n'existe

1 Il y a toutefois quelques appendices, qui sont publiés, comme d'habitude, avec le volume 1 des Journaux de l'Assemblée Législative du Québec. Le fait que les Documents de la Session ne commencent qu'avec la session 1869 provoquera d'ailleurs un décalage entre la numérotation des volumes de Journaux et celle des Documents et cela, jusqu'en 1872 inclusivement. Pour rétablir le parallélisme entre la numérotation des deux séries, on sauta un numéro de volume, dans la série des Documents de la Session: On passa ainsi du vol. 5 (1872) au vol. 7 (1873-1874).

2 Il existe, à notre connaissance, un seul index, pour les Appendices et les Documents de la Session du Canada de 1841 à 1866, celui de Patricia Damphouse, The Legislative Assembly of the Province of Canada: an Index to Journal Appendices and Sessional Papers, 184I-1866 (London, Ont., E. Phelps, 1974). Il s'agit là d'un instrument très utile, et malheureusement pas assez connu; il diffère cependant de l'inventaire que nous avons effectué, en ce sens qu'il ne rend pas compte, comme nous tentons de le faire de la structure et du contenu de chacun des documents. 
pas d'index pour chacun des volumes, et on ne dispose d'aucun instrument qui permette de rendre compte, en une seule opération, du contenu de l'ensemble de la série. Par ailleurs, les tables des matières de chaque volume ne constituent pas un instrument de repérage suffisamment fin pour répondre aux besoins des chercheurs.

C'est pour obvier à cet inconvénient que nous avons entrepris depuis bientôt un an, la confection d'un inventaire descriptif des Documents de la Session, inventaire qui sera terminé en mai 1978. Nous exposons brièvement, dans les pages qui suivent, les modalités de réalisation de cet inventaire et les conditions de son utilisation. Auparavant, quelques mots pour expliquer comment se présente la série de documents, de 1841 à 1936.

Les Documents de la Session sont préparés par divers services publics ou para-publics, soit sur l'ordre du lieutenant-gouverneur en Conseil, soit encore conformément aux lois et règlements en vigueur; les rapports annuels des ministères, ou encore des hôpitaux et asiles, par exemple, n'ont pas à être demandés; ils sont produits obligatoirement. Parmi les documents ainsi préparés, un certain nombre, choisis par la Comité des Impressions (Comité conjoint des deux Chambres), sont publiés et forment la série des Documents de la Session.

La liste complète des documents préparés à l'usage des parlementaires figure à la table des matières des Journaux de l'Assemblée Législative, sous la rubrique Comptes et papiers jusqu'en 1852-1853 inclusivement, puis sous la rubrique Documents, à partir de 1854 . Outre le titre des documents, cette liste donne leur référence dans les Documents de la Session («Appendice A.A.», par exemple; ou: «Document no 27»). Il faut noter cependant que le fait qu'un document soit énuméré dans cette liste ne signifie pas nécessairement qu'il a été publié. En effet, à partir de 1861, il arrive de plus en plus fréquemment qu'un document soit mis à la disposition des par- 
lementaires,--sous forme manuscrite, et ne soit pas publié ${ }^{3}$. Dans ces cas, seuls les titres des documents apparaissent dans les volumes publiés, immédiatement suivis de la mention: "Conformément à la recommandation du Comité des Impressions, ce document ne doit pas être imprimé».

Les volumes publiés des Documents de la Session ne comprennent donc qu'une partie des documents effectivement mis à la disposition des parlementaires au cours de la session. Il s'agit évidemment des plus importants, et ordinairement, toutes les grandes séries sont publiées; il arrive cependant que certaines ne le soient que de façon intermittente: c'est le cas par exemple des statistiques de l'état civil, ou encore des statistiques municipales.

Les documents semblent avoir été assemblés sans souci d'un quelconque ordre logique. À partir de 1886 toutefois, on constate qu'en règle générale, les rapports des ministères sont regroupés dans les premiers tomes, tandis que les réponses aux adresses de I'Ássembiée sont placées dans le dernier. Enfin, les documents eux-mêmes manquent d'homogénéité, et cela, surtout au $19^{\mathrm{e}}$ siècle: on retrouve ainsi, à l'intérieur d'un même document des éléments qui souvent n'ont rien de commun.

Ces quelques inconvénients n'enlèvent rien à la valeur de cette série documentaire unique. Ils laissent cependant soupçonner les difficultés inhérentes à toute entreprise visant à rendre plus accessible une masse de documents aussi hétéroclites.

Le projet a pour objectif la création d'un inventaire détaillé des Documents de la Session - y compris les Appendices aux Journaux de l'Assemblée Législative - pour la période 1841-1936. À partir de 1867, seule la série du Québec a été inventoriée.

3 Le plus souvent, ces documents non publiés n'ont qu'un intérêt très limité, sur le plan national: ils ne concernent qu'un individu, ou une région, et non l'ensemble du Québec. Ces documents subsistent encore, pour la plupart, soit aux archives Publiques du Canada, pour la période pré-confédération (R.G. 14), soit aux Archives de l'Assemblée Nationale du Québec, pour la période 1867-1936. Il existe une copie sur microfilm de cette dernière série à la Bibliothèque de la Législature. 
Lorsqu'il sera terminé, cet inventaire se présentera sous la forme de deux fichiers; un fichier descriptif et un fichier de référence.

Le fichier descriptif. Les fiches descriptives sont des fiches numérotées, sur lesquelles sont portés tous les renseignements concernant les documents : référence, titre, contenu. Tout document qui a été publié fait l'objet d'une ou plusieurs fiches descriptives (Annexe I). Ces fiches sont conçues de façon à donner très rapidement un aperçu du document. En effet, outre la référence et le titre, la fiche descriptive donne deux autres types d'information:

- l'importance quantitative du document, c'est-à-dire le nombre de pages,

- une description abrégée du contenu; il est rare en effet que le titre seul soit suffisant pour décrire la matière d'un document. On utilise donc, pour le compléter, des descripteurs (mots-clés, vedettes-sujets) qui remplissent deux fonctions: 1) d'abord, permettre de rattacher un document quelconque à un ensemble de documents traitant de la même catégorie de sujets; c'est le rôle du descripteur général. On trouvera en Annexe II une liste des descripteurs généraux qui ont été utilisés; 2) en outre, on utilise des descripteurs particuliers qui ont pour fonction de donner un aperçu des questions traitées dans les documents.

Ces fiches descriptives sont classées par ordre alphabétique des descripteurs généraux, et, à l'intérieur de chaque descripteur général, par ordre chronologique.

Le fichier de référence. La nécessité du fichier de référence provient de ce que la fiche descriptive n'existe qu'en un seul exemplaire et ne peut donc être classée que sous un seul sujet; or, il est très fréquent qu'un même document contienne des renseignements sur plus d'un sujet. Il fallait donc créer un second fichier, pour renvoyer l'utilisateur à tous les documents recélant des informations sur le sujet qui l'intéresse. Les fiches de référence ne contiennent qu'un descripteur (par exemple: Agriculture-Technologie) et des numéros, ceux-ci renvoyant l'utilisateur aux fiches descriptives. En consultant toutes les fiches correspondant à ces numéros, l'utilisateur est assuré d'avoir relevé toutes les références sur son sujet. 
Étant donné l'ampleur du travail impliqué dans la confection de ce fichier, nous avons décidé de ne faire de fiches de référence que pour certains sujets que nous jugions particulièrement importants: on trouvera en Annexe III la liste des sujets qui ont été retenus. Il s'agit, comme on pourra le constater aisément, d'une liste assez complète pour répondre aux besoins de la majorité des chercheurs.

Les utilisateurs qui s'intéresseraient à un sujet non compris dans la liste des sujets retenus trouveront quand même leur profit à utiliser l'Inventaire, puisque tout document, quelle qu'en soit la nature, fait l'objet d'une fiche descriptive. Ainsi, un chercheur s'intéressant aux relations extérieures (sujet non retenu pour le fichier de référence) trouvera, dans le fichier descriptif, les références aux documents traitant directement et principalement de ce sujet à la rubrique: RELATIONS EXTÉRIEURES.

Comme cet inventaire constitue un instrument de travail permettant un accès pius rapide à i’information contenue dans ies $\bar{D} o c u$ ments de la Session, nous avons jugé bon d'en informer les chercheurs intéressés. De fait, les annexes illustrent en partie la variété et la richesse du contenu de cette série de documents et l'importance de l'inventaire qui en a été fait. Par ailleurs un document décrivant en détail les procédures suivies dans l'établissement des fiches d'inventaire est disponible sur demande.

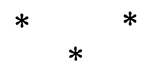


ANNEXE I

Exemples de fiches descriptives

1849 - A.J.A.L.P.C., Y.Y.Y.Y.

Comptes de la seigneurie de LAUZON, depuis l'appropriation par la Couronne jusqu'au 20-12-1848.

12 pages

Lods et ventes. Cens et rentes. Loyer des moulins. Affermage des terres et autres propriétés. Conditions des moulins et autres bâtiments.

1854-1855 - A.J.A.L.P.C., F.F.F.F.

Tableaux des mariages, baptêmes et sépultures dans les districts de Québec, Montréal, Trois-Rivières et Gaspé pour l'année 1854.

35 pages

Par comtés, par paroisses, par dénomination religieuse. Augmentation et diminution. Tableau supplémentaire pour le district de Montréal pour les années 1851, 1852, 1853. 


\section{ANNEXE II}

Liste des descripteurs généraux

A

Administration coloniale

Affaires culturelles

Affaires municipales

Affaires sociales

Agriculture

Amérindiens

Armée

Archives

Associations volontaires

Assurance

$\bar{B}$

Bibliothèque

Bois

Bureaux d'enregistrement

C

Chasse (et pêche)

Clergé

Colonisation

Commerce

Communications

Compagnie de la B. d'Hudson

Comptes publics

Concession des terres

Conditions de travail

Confédération

Crédit

D

Divers

Droit civil

Douanes
E

Édifices publics

Éducation

Élections

Émeutes

Énergie

F

Faillites

Fléaux

Fonction publique

Forges du St-Maurice

Frontière

G

Géographie

I

Industrie Innovations

Institutions financières

Institutions religieuses

J

Justice

L

Législature

M

Météorologie

Milice 
Mines

Monnaie

$\mathbf{P}$

Pêcheries

Population

$\mathrm{R}$

Rébellion de 1837-1838

Régime seigneurial

Relations extérieures

Relations fédérales-provinciales
S

Services publics

Sociétés par actions
$\mathrm{T}$

Taxe

Tempérance

Terres de la Couronne

Travaux publics. 


\section{ANNEXE III \\ Liste des sujets pour lesquels on fera des fiches de référence}

1. AGRICULTURE.

1.0 Général.

1.1 Concession des terres.

1.2 Colonisation (aussi inclus: chemins de colonisation).

1.3 Technologie.

1.4 Productions.

1.5 Industrie laitière.

1.6 Pédologie (i.e. composition des sols).

1.7 Élevage (vaches et autres).

1.8 Main-d'œuvre.

1.9 Marché.

1.10 Activités subsidiaires (connexes...).

1.11 Conditions financières.

1.12 Associations.

1.13 Éducation.

2. AMÉRINDIENS.

3. ASSURANCES.

4. BOIS.

4.0 Général.

4.1 Concession des limites forestières.

4.2 Sortes; coupes; licences.

4.3 Entrepreneurs et compagnies.

4.4 Chantiers.

4.5 Scieries.

4.6 Moulins.

4.7 Commerce et transport.

4.8 Rapports agro-forestiers.

5. BUREAUX D'ENREGISTREMENT.

6. CADASTRE.

7. COMMERCE.

7.0 Général.

7.1 Intérieur.

7.2 Extérieur. 


\section{COMMUNICATIONS.}

8.0 Général.

8.1 Navigation (aussi inclus: écoles de navigation).

8.2 Chemins de fer.

8.3 Routes (aussi inclus: chemins de colonisation; ponts...).

8.4 Postes.

8.5 Télégraphe.

8.6 Téléphone.

9. CONJONCTURE ÉCONOMIQUE.

11. INTEMPÉRANCE (aussi inclus: licences d'auberge...).

12. JUSTICE.

12.0 Général (actes, règlements, rapports statistiques, construction de palais de justice...).

12.1 Administration (cours, juges, dépôts, avocats, salaires, timbres, termes des cours...).

12.2 Système judiciaire (accusations, jugements, procès, amendes, sommations, writs, jurés...).

12.3 Police.

12.4 Système pénitentiaire.

13. POPULATION.

13.0 Général.

13.1 Mouvement de la population (N.M.D.).

13.2 Structure de la population (recensements, distribution ethnique...).

13.3 Mouvements migratoires.

14. SANTÉ.

14.0 Général.

14.1 Établissements.

14.2 Personnel médical.

14.3 Prévention.

14.4 Médecine illégale.

14.5 Maladies; épidémies.

14.6 Soins; remèdes.

14.7 Hygiène publique.

15. RÉGIME SEIGNEURIAL. 
16. INDUSTRIES.

16.0 Général.

16.1 Hydro-électricité.

16.2 Mines.

16.3 Autres industries.

16.4 Salaires et conditions de travail.

16.5 Syndicats et associations.

16.6 Main-d'œuvre.

16.7 Villes-compagnies.

17. ÉDUCATION.

18. AFFAIRES MUNICIPALES. 